scanning tunneling microscopy (STM), the researchers examined "metallic" zigzag $(\theta=0)$ and armchair nanotubes $(\theta=30)$. While it has been postulated that curvature effects alter the overlap of $\pi$ electron wave functions creating small energy gaps in "metallic" zigzag and chiral $(0<\theta<30)$ SWNTs at the Fermi energy $\left(E_{\mathrm{f}}\right)$ and that intertube interactions can break the rotational symmetry of armchair SWNTs, there has been no experimental verification of these points. STM measurements were carried out in ultrahigh vacuum at $\sim 5 \mathrm{~K}$ on SWNT samples supported on $\mathrm{Au}(111)$ substrates. The SWNT indices $(n, m)$, which are related to the tube diameter and chiral angle and are typically used to describe nanotubes, were assigned from the STM images. Tunneling conductance data were obtained by tunneling spectroscopy simultaneously with the STM images, and the DOS was calculated using a $\pi$-only tight-binding calculation for the assigned indices. For the "metallic" zigzag structures, the researchers' spectroscopy data showed previously unobservable gaplike structures at $E_{\mathrm{f}}$ that demonstrate that these tubes are small-gap semicon- ductors and not metals. As predicted, the gap magnitude was seen to depend inversely on the square of tube radius indicating that these gaps arise from curvature in the graphene sheet. Armchair nanotube experiments were performed both on a SWNT in a bundle and on an isolated tube on a $\mathrm{Au}(111)$ surface. Tunneling spectra on the isolated nanotube did not show any gaps but rather behaved as true metals as expected because of the crossing of $\pi$ and $\pi^{*}$ bands at $E_{\mathrm{f}}$. However, measurements on the nanotube in a bundle showed a gaplike feature that arose due to interactions between multiple tubes; this feature, termed a pseudogap, differs from the curvature-induced gaps seen in the zigzag SWNTs, because the DOS are suppressed but not reduced entirely to zero at $E_{\mathrm{f}}$. The measurements being carried out by Ouyang and co-workers will help clarify the nature of nanotube electronic DOS near the Fermi level and thus increase the understanding of electrical transport through "metallic" SWNTs.

"The absence of gaps and pseudogaps in isolated armchair SWNTs," said Ouyang, "indicates that through control of the local environment of these tubes, it will be possible to maximize their conductivity, possibly as interconnects in nanoelectronics."

STEFFEN K. KALDOR

\section{Chalcogenide Glasses Show Photoinduced Second-Harmonic Generation}

Researchers at the Shanghai Institute of Optics and Fine Mechanics, the Japan Science and Technology Corp., and Kyoto University have observed photoinduced stable second-harmonic generation (SHG) in chalcogenide glasses. As reported in the June 15 issue of Optics Letters, J. Qiu, J. Si, and K. Hirao optically encoded SHG in $20 \mathrm{Ge}-20 \mathrm{As}-60 \mathrm{~S}$ (mol\%) glass with nanosecond laser pulses. The glass exhibits excellent photoinduced SHG conversion efficiency and stability compared to tellurite and bismuth oxide glasses.

The glass was prepared by heating a 50 -g mixture of Ge, As, and S in a silica ampule in a rocking electric furnace at $800^{\circ} \mathrm{C}$ for $2 \mathrm{~h}$ under $0.1-\mathrm{Pa}$ vacuum. The sample was quenched to room temperature and annealed at the glass-transition

$D$ papers published daily $D$ highest impact factor in the field $D$ electronic submission and publication
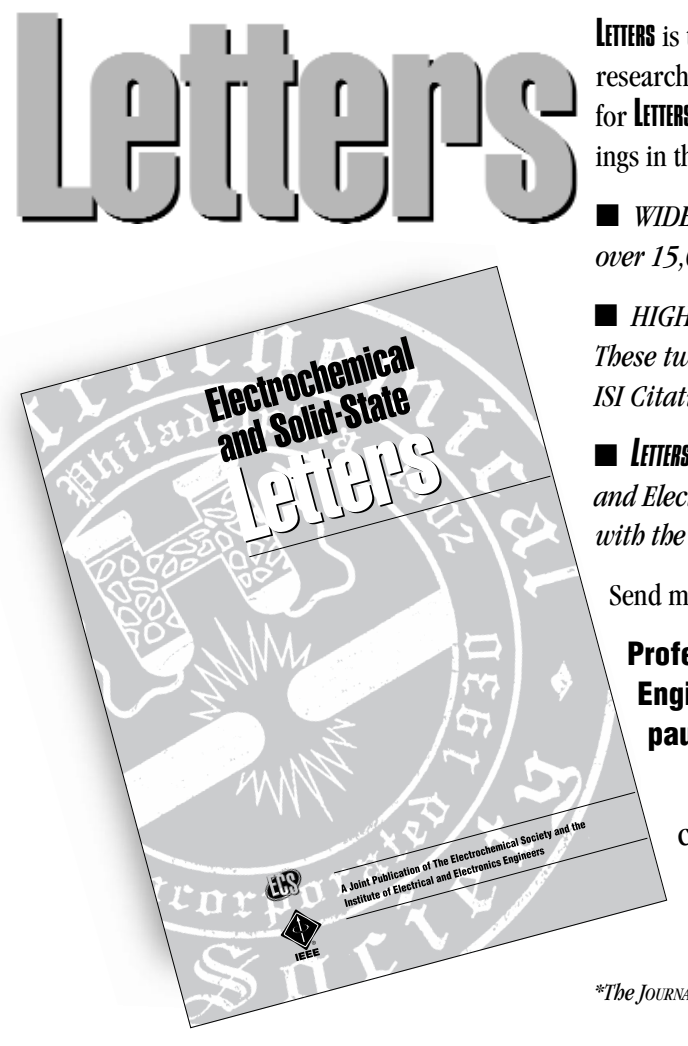

LFTERS is the first rapid-publication electronic-first journal dedicated to covering the leading edge of research and development in the fields of electrochemical and solid-state sciences. Articles accepted for LETाERs are published daily on the web, ensuring rapid dissemination of the latest research and findings in these fields. Now in its fourth year, LFTER offers valuable benefits to its authors:

WIDE CIRCULATION: LETIER has the widest distribution of any letters journal in the field, with 15,000 individuals, institutions and libraries.

HIGH QUALITY: IfTERs is a companion publication to the JOURNAL OF THE ELECTROCHEMICAL SOCIETY. These two publications had the highest impact factors in electrochemistry, according to the latest citation Index rankings (1999)."

IFTIER is a joint publication of The Electrochemical Society (ECS) and the Institute of Electrical nd Electronics Engineers (IEEE) Electron Devices Society (EDS), and is published in cooperation ith the American Institutue of Physics (AIP).

end manuscripts electronically or on paper to:

Professor Paul Kohl, Georgia Institute of Technology, School of Chemical Engineering, 778 Atlantic Drive, Atlanta, GA 30332-0100 USA, paul.kohl@che.gatech.edu

To learn more, contact ECS by calling 609737 1902, or contact us via email: publications@electrochem.org; or visit the lFtifRs home page at: www3.electrochem.org/letters/html

Circle No. 12 on Inside Back Cover 
temperature for $1 \mathrm{~h}$. It was then ground and polished to $0.5 \mathrm{~mm}$ thick.

SHG was obtained by pulses of $3.6 \mathrm{~ns}$ at $1.064 \mu \mathrm{m}$ (beam $\omega$ ) and $3 \mathrm{~ns}$ at $532 \mathrm{~nm}$ (beam $2 \omega$ ) from a Q-switched Nd:YAG laser. The samples were prepared with both beam $\omega$ and beam $2 \omega$ incident. The samples were probed with beam $2 \omega$ blocked. Behind the sample, two heatabsorbing filters and a 532-nm bandpass filter allowed a photomultiplier to detect only the SHG signal with beam $2 \omega$ blocked. The signal was averaged by an oscilloscope.

The intensity of SHG, normalized by the maximum intensity of second-harmonic waves from 1-mm-thick Y-cut quartz, increased rapidly and saturated after $50 \mathrm{~min}$. The maximum intensity was seven times larger than that of the quartz and four orders of magnitude larger than that of $15 \mathrm{Nb}_{2} \mathrm{O}_{5}-85 \mathrm{TeO}_{2}$ under the same optical poling conditions. As-made samples showed no SHG.

The signal from the chalcogenide glass was stable, unlike other glasses. The signal from $\mathrm{Bi}_{2} \mathrm{O}_{8}$-based glasses decreased to $80 \%$ after $10 \mathrm{~min}$. For $15 \mathrm{Nb}_{2} \mathrm{O}_{5}-85 \mathrm{TeO}_{2}$, the signal decreased to $5 \%$ after $10 \mathrm{~min}$.

The researchers suggest that the nonlinear coherent field of $\omega+2 \omega$ creates free electrons that are trapped by active sites in the glass, which breaks the inversion symmetry of the microstructure and allows SHG.

Selecting the proper glass composition could increase the conversion efficiency and stability of the SHG, the researchers said. They believe that with their technique "it is possible to achieve practically useful nonlinear optical frequency-conversion devices with a chalcogenide glass fiber."

ELIZABETH A. SHACK

\section{Nonvolatile Memory Observed in Chromium-Doped $\mathrm{SrTiO}_{3}$ Single Crystals}

Materials that exhibit reversible resistive switching are potential candidates for random-access memory (RAM). Various metal-insulator-metal-oxide structures display switching and show memory retention of more than 18 months. Y. Watanabe and colleagues, members of the IBM research team at Zurich Research Laboratory, examined single crystals of one such oxide, $\mathrm{Cr}$-doped $\mathrm{SrTiO}_{3}$. Using these single crystals as a model system, the researchers determined that microstructural defects in the thin films are not a significant contribution to the switching effect. Instead, the researchers conclude that a bulk electronic change is necessary for memory switching to occur.

As reported in the June 4 issue of
Applied Physics Letters, at $4 \mathrm{~K}$ the crystal has an initial resistance of $1 \mathrm{G} \Omega$ up to $100 \mathrm{~V}$. Sweeping to $200 \mathrm{~V}$ causes a drop in resistance leading to a hysteretic currentvoltage characteristic. Stressing the crystal with pulsed or dc voltage causes an additional resistance drop by several orders of magnitude. This creates a conductive state which allows for memory switching. By applying a positive voltage pulse, the oxide is switched to a low-impedance state with a resistance of $500 \Omega$. Applying a negative voltage erases the "information" written to the device and switches the oxide back to a high-impedance state with a resistance of $5000 \Omega$. This effect is also seen at room temperature. Once the conductive state is established, the switching behavior of the single crystal is reproducible. After more than $10^{5}$ read pulses, no change in the readout signal was seen, indicating that these single-crystal oxides have the potential to be alternative nonvolatile RAM.

The conductive state is created by exposing the insulating crystal to high voltages. During temperature cycling experiments, the low state tended to change to the high state. This suggests to the researchers that the conductive state originates from an excessive amount of injected carriers. This was verified by $\mathrm{Cr}$-doped $\mathrm{SrZrO}_{3}$ thin-film experiments. A good scaling of the current versus electrode area from $0.5 \mathrm{~mm}$ square to $100 \mathrm{~mm}$ square is observed, which indicates to the researchers that this is not a defect-dominated process. The researchers said that the high resistance of the single crystal at low voltage confirms that the bulk determines the current flow across the insulator and that the transition to the conductive state originates from a change in the bulk property.

\section{JENNIFER L. BURRIS}

\section{$(\mathrm{Pb}, \mathrm{Sr}) \mathrm{TiO}_{2}$ Thin Films Prepared by LSMCD as Potential DRAM Materials}

Researchers at the Department of Chemical Engineering at the Korean Advanced Institute of Science and Technology and Samsung Electronics Co. have prepared high-quality thin films of $(\mathrm{Pb}, \mathrm{Sr}) \mathrm{TiO}_{2}$ (PST) on Si wafers and Pt-coated Si wafers by liquid source misted chemical deposition (LSMCD). Seong I. Woo and co-workers found that LSMCD produced high-quality films that exhibited a high dielectric constant, good paraelectricity, and low dielectric loss after annealing at a relatively low temperature of $550^{\circ} \mathrm{C}$. According to the researchers, these properties make the "PST thin film a promising material for ULSI-DRAM [ultralarge-scale integration dynamic random-access memory] capaci- tors and other microelectronic device applications."

As reported in the May 2001 issue of Chemistry of Materials, the researchers used lead acetate, strontium acetate, and titanium isopropoxide as the metallic precursors for the film. A solution of the precursors in acetic acid and 1-butanol was misted using an ultrasonic nebulizer and the mist transported to the deposition chamber by a stream of argon. The films were grown on $\mathrm{Si}(100)$ wafers and $\mathrm{Si}(100)$ wafers coated with $100 \mathrm{~nm}$ of Pt. The films were baked at $400^{\circ} \mathrm{C}$ for $10 \mathrm{~min}$ and annealed between $500^{\circ} \mathrm{C}$ and $700^{\circ} \mathrm{C}$ for $5 \mathrm{~min}$. All heat treatment was performed in air. Films with thicknesses of 60,90 , and $130 \mathrm{~nm}$ were prepared.

Scanning electron micrographs of the PST films revealed smooth, dense, and uniform surfaces devoid of any gaps or cracks. X-ray diffraction and Auger electron spectroscopy also showed that the polycrystalline film exhibited uniform grain size and composition throughout its thickness. Wavelength dispersive spectroscopy was used to confirm that the composition of the film was identical to the composition of the precursor solution used for misting.

Measuring the capacitance and current as functions of applied voltage in the PST showed that the films exhibited paraelectric behavior, low leakage currents, and high dielectric constants. The researchers predict that the leakage current and paraelectricity in the films could be further improved by postannealing under oxygen and reducing the amount of $\mathrm{Pb}$ in the films, respectively. The electrical properties measured for the PST films compare favorably to those of $(\mathrm{Ba}, \mathrm{Sr}) \mathrm{TiO}_{2}$ (BST) films, which are considered the most promising material for ULSI-DRAM capacitors. The BST films, however, must be annealed at $700^{\circ} \mathrm{C}$ while PST films can be annealed at $550^{\circ} \mathrm{C}$. This reduction of annealing temperature is important in electronics fabrication because high annealing temperatures can damage sensitive electronic components.

GREGORY KHITROV

\section{Self-Assembled Diblock Copolymers Applied in Semiconductor Capacitor Fabrication}

Advances in microfabrication technologies over the last few years have allowed reductions in the physical size of integratedcircuit (IC) components. Shrinking the size of the memory cell has facilitated dynamic random-access memory (DRAM) chips with near-gigabit storage capacities while maintaining similar sizes to their 1-MB 